\title{
Poland's anomaly - A case report
}

P. S.V Devi ${ }^{1}$, C.K.Reddy ${ }^{2}$, N. Satyanarayana ${ }^{3}$, P. Sunitha ${ }^{4}$, N.Reddy ${ }^{5}$, M.J. Phukon ${ }^{6}$. A.S. Ram ${ }^{7}$.

${ }^{1}$ Profesor and Head, ${ }^{2,6}$ Associate professor, ${ }^{6}$ P.G., Department of Anatomy Prathima Institute of Medical Sciences, Karimnagar, Andhra Pradesh, India, ${ }^{3}$ Assistant Professor in Anatomy, ${ }^{4}$ Lecturer in Physiology, College of Medical Sciences, Bharatpur, Chitwan, Nepal, ${ }^{5}$ Associate professor Departmetn of Anatomy, CAIMS,Kariminager,India.

\begin{abstract}
A 12 year old boy was admitted in the medical ward, Prathima Institute of Medical Sciences hospital, Karimnagar, with severe dyspnoea of one week duration. During the clinical examination, besides the auscultatory findings, it was observed that the boy was a typical case of Poland's anomaly, characterized by deficiency of the sterno-costal part of Pectoralis major and entire pectoralis minor, minor associated with hypoplastic, thenar and hypothenar muscles. Considering the rarity involved in finding such a sporadic, congenital but non fatal medical condition, knowledge of musculoskeletal anomalies is important for clinicians, orthopedics and plastic surgeons.
\end{abstract}

Keywords: Poland's anomaly, deficiency, musculoskeletal anomalies.

\section{Introduction}

Poland's anomaly is a congenital musculoskeletal abnormality characterized by deficiency of the sternocostal part of pectoralis major and entire pectoralis minor, in association with ipsilateral abnormalities of the limb of various degrees ranging from syndactyly to hypoplasia of entire limb musculature and bones. ${ }^{1,2}$ In addition, many other systemic, organic or integumentary anomalies (renal, aplasia/ agenesis, gonadal dysgenesis, dextrocardia), thrombocytopenia, leukemia, spherocytosis and lymphomas and syndromes like Mobius and Klippelfiels might also be involved. ${ }^{3,4}$

\section{Case report}

A 12 year old boy admitted in medical ward, Prathima Institute of Medical Sciences hospital,

Correspondences: Dr. N. Satyanarayana.

E-mail: satyam_n19@yahoo.com
Kariminager, had presented with severe dysponea of seven days duration. A deep depression in the upper right chest with a bulging in that area on each act of respiration was observed. The right nipple and areola were hypoplastic.

They referred the case to the Department of clinical Anatomy to rule out musculoskeletal abnormality of chest. On palpation, it was observed that pectoralis major and minor, anterior ends of $2^{\text {nd }}$ to $4^{\text {th }}$ ribs and the corresponding costal cartilages and intercostal muscles were absent. The right lateral border of sternum was everted, right clavicle was shorter than the left. Upper parts of ipsilateral serratus anterior and external oblique were absent. Latissimus dorsi was very hypoplastic (Fig.1, 2).

On left side of the chest, no muscular variation was detected. Thenar and Hypothenar muscles were hypoplastic and as a result, fine actions of hand and fingers could not be performed (Fig 3). There were no 
digital anomalies observed. Length of both limbs were equal. No other organic or systemic abnormality was found on investigations. The intelligence and growth were normal.

Family history suggested no hereditary or familial case. We were informed that the patient had three female siblings and none of them had this sort of clinical condition. The patient's mother was reported to be addicted to tobacco and aspirin consumption and had a couple of threatened abortions in first trimester.

No consanguinity among the parents was involved, the delivery and the subsequent milestones of the child were reported to be normal. Even though the defect was observed during the neonatal period itself, it was never reported to any hospital. A history of frequent nasal allergies and respiratory infections was reported.

\section{Discussion}

Poland's anomaly is a rare entity with average incidence of one in 30,000 births. In the year 1841, Sir Alfred Poland reported the absence of pectoralis major in a cadaver he was dissecting on, when he was a medical student in Guy's hospital, United Kingdom. This condition associated with other muscle and nerve abnormalities in the anterior chest wall and ipsilateral synbrachydactyly was named as "Pectoral Deficiency Syndrome". ${ }^{1}$ A few more such similar incidences,often associated with ipsilateral syndactyly were later reported and the medical condition was named after its original discoverer as "Poland's Syndrome". 2,5,6

Later on, the mid-phallangeal hypoplasia, which was consistently found to be involved, was taken as the clinical criterion for Poland's anomaly. 7,8,9,10

Bing observed abnormalities in the posterior shoulder region, some with springel deformity, in 9 out of 102 cases of pectoralis major deficiency. ${ }^{11}$
Scapular abnormalities including winging of scapula along with absence of upper part of serratus anterior were found with Poland's anomaly. ${ }^{12}$

Though syndactyly and synbrachydactyly were observed to be associated more with pectoral deficiency, the posterior shoulder girdle muscles might not have been observed in all the cases. It has been suggested that the aetiology of Poland's anomaly is unclear; the condition is caused by disruption of the arterial blood supply to the subclavian vessels during the sixth and seventh week of embryonic life, or by disruption of lateral plate mesoderm 2 to 4 weeks after fertilization. ${ }^{13}$

In the present case, the wide spread musculoskeletal abnormality of anterior chest wall, posterior shoulder girdle and the upper limb may be attributed to the disruption of the arterial blood supply to the subclavian vessels during the sixth and seventh week of embryonic life or by disruption of lateral plate mesoderm 2 to 4 weeks after fertilization.

Recent embryological studies indicated that the release of signaling molecules from the neural crest cells close to the developing parts appear to be responsible to differentiate the embryonic mesenchyme, the somatopleuric mesoderm into skin, fascia, vessels, nerves, muscles, cartilage and bone, intermediate mesoderm into genitourinary system and para-axial mesoderm in to vertebrae and post axial muscles. Any delay or deficiency in releasing the signaling molecules may produce the various defects on the same side in proportion to the severity, particularly in the first trimester. $^{14}$

\section{Conclusion}

Whatever may be the cause producing the defect - "Poland's anomaly", the patient must be thoroughly 
investigated for any associated internal or external defects by routine and modern investigations. Guidance should be given for permanent cure of the defect or deformity by plastic surgery and rib grafting by orthopedic surgeons. Any defect of the fingers should be treated by plastic surgery as early as first year after birth. Any chest wall abnormality may be treated by plastic surgery or bone grafting or by prosthesis after reaching the adolescence.

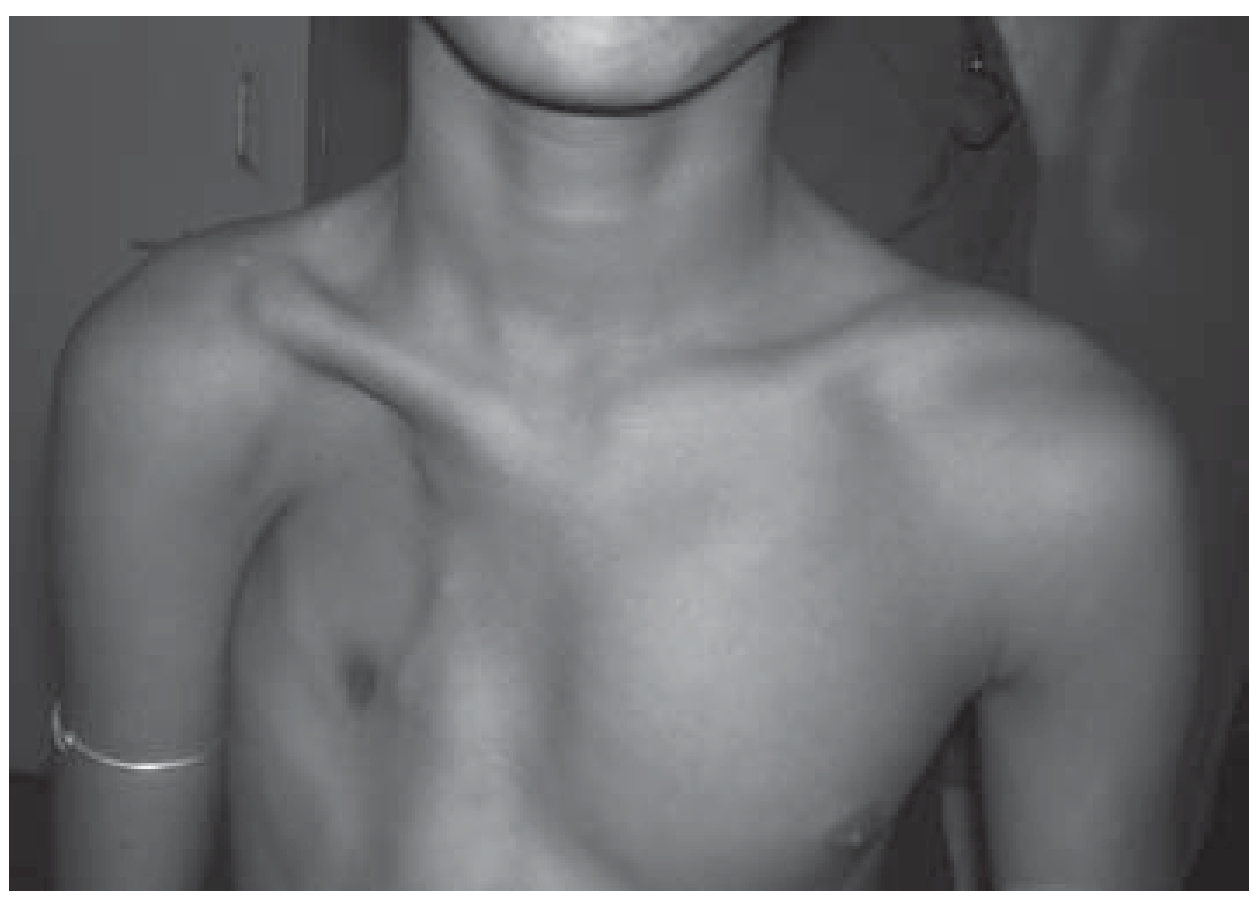

Fig.1.Showing a deep depression on right side of chest, absence of right side pectoralis major, minor muscles, intercostal muscles, serratus anterior, external oblique muscle, anterior ends of $2^{\text {nd }}$ to $4^{\text {th }}$ ribs and corresponding costal cartilages.

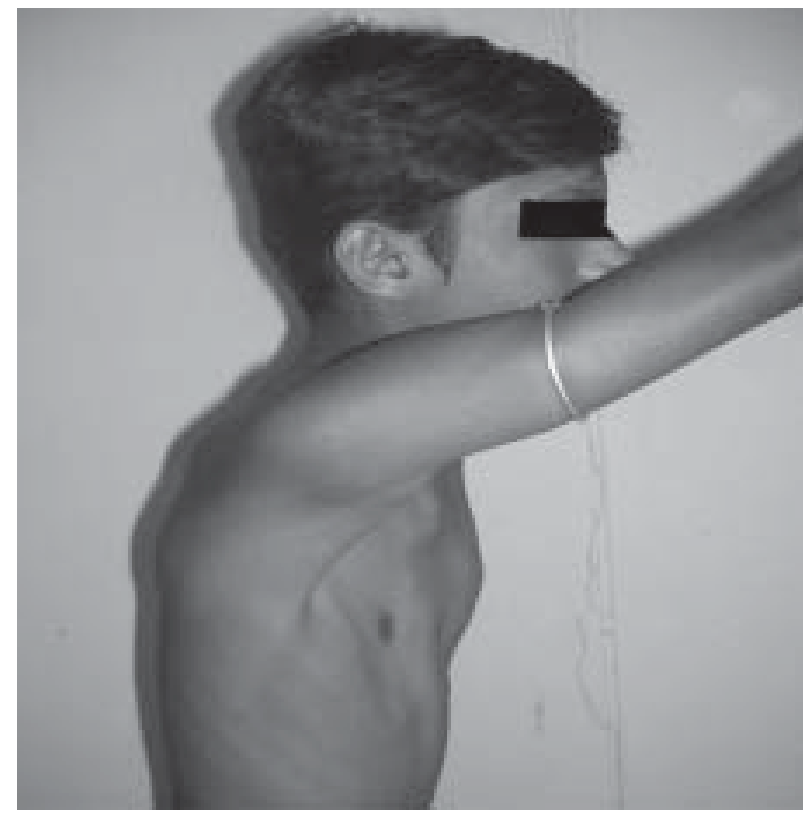

Fig.2. Showing hypoplastic right latissimus dorsi muscle, absence of right serratus anterior muscle.

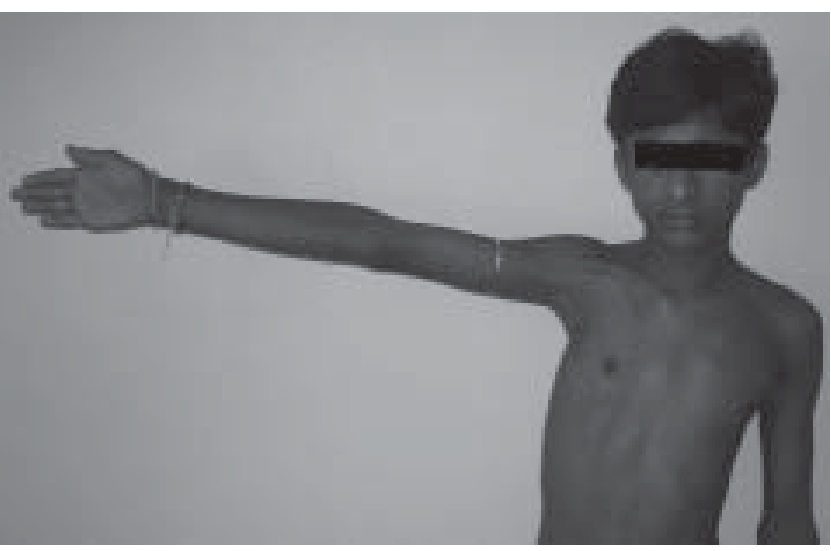

Fig.3. Showing hypoplastic right thenar and hypothenar muscles of palm. 
P. S.V Devi et al, Poland's anomaly - A case report

\section{References}

1. A. Poland . Deficiency of the pectoral muscles. Guys Hosp Rep. 1841; 12:191-3.

2. J.W. Mace, J.M. Kaplan, J.E. Schanberger, et al Poland's syndrome. Report of seven cases and review of the literature. Clin Pediatr 1972; 11:98-102.

3. R.A. Mille, D.A. Miller Letter Congenital absence of the pectoralis major muscle with acute lymphoblastic leukemia and genitourinary anomalies. J Pediatr 1975; 87:146-7.

4. M.M.Ravitch, Poland's sydrome--a study of an eponym. Plast Reconstr Surg 1977; 9: 508-12.

5. J.B. Brown, F. McDowell. Syndactylism with absence of the pectoralis major. Surgery 1940; 7:599-601.

6. P. Baudinne, G.L.Bovy, A. Wasterlain Acase of Poland's syndrome. Acta Paediatr Belg 1967; 21:407-10.

7. J.L. Frias, A.H. Felman. Absence of the pectoralis major, with ipsilateral aplasia of the radius, thumb, hemi diaphragm and lung: an extreme expression of poland anomaly? Birth Defects Orig Artic Ser 1974; 10:55-9.

8. D.W.Smith Recognizable patterns of human malformation. Major Probl Clin Pediatr 1976; 1-497.

9. S.A.Temtamy, V.A. McKusick The genetics of hand malformations. Birth Defects Orig Artic Ser 1978; 14:1619.

10. R.K. Beals, S. Crawford. Congenital absence of the pectoral muscles. A review of twenty-five patients. Clin Orthop Relat Res 1976; 166-1.

11. R. Bing, Ueberangeborene. Muskeldefecte Virchow's Archive 1902; 170:175-228.

12. D.C. Ireland, N. Takayama, A.E. Flatt Poland's syndrome. J Bone Joint Surg Am 1976; 58:52-8

13. S. Stranding, H. Ellis , J.C. Healy et al; Pectoral Girdle, Shoulder Region and Axilla. Gray's Anatomy. $39^{\text {th }}$ ed, London Churchill Livingstone. 2005; 835.

14. T.W. Sadler Muscular System. Longman's Medical Embryology 2006; 0:148. 